\title{
Energy Deposition by High Energy Particle Beam Radiation
}

\section{John R Sabin*}

Department of Physics, University of Florida, Florida, USA

\section{Review Article}

Volume 2 Issue 1

Received Date: May 26, 2018

*Corresponding author: John R. Sabin, Department of Physics, University of Florida, Gainesville, Florida, USA, Email: sabin@qtp.ufl.edu

\section{Abstract}

The mean excitation energies of some atoms and some ions are discussed. In any series, the largest changes in mean excitation energy for single electron occupation number changes occur when the change involves different sub-orbital's.

Keywords: Tumor treatment; X-ray beam; Heavy ion beam; Photon beam; Absorb energy; Large energy deposition

\section{Introduction}

Over the past several decades, energy deposition into materials has become ever more useful, ranging from electronics manufacture to tumor treatment. In recent times, a fast ion beam, frequently a proton beam is impinged on a material. The reason for this is that the maximum in the relative dose (the amount of energy deposited) as a function of the depth from a sample surface from an ion beam can be selected much more specifically than that from a photon beam such as x-rays, resulting in the ability to irradiate a particular area, for an ion beam, while for a photon beam this is not possible, as shown in Figure 1 [1]. Thus, in the case of particles, there is less damage to the surrounding area in the case of a proton beam than in the case of an x-ray beam. This is particularly important for treatment of tumors.
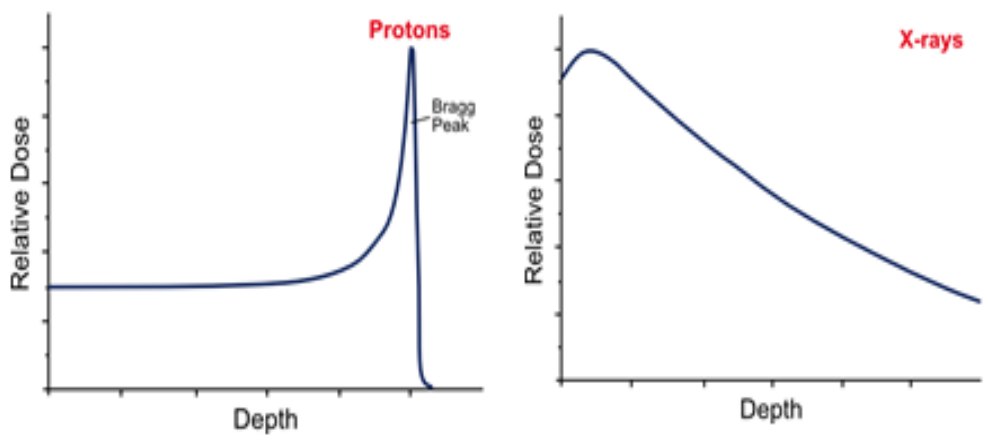

Figure 1: Relative dose vs. depth from sample surface for a heavy ion beam (protons) and for a photon beam (x-rays) in ordinary matter.

The translational energy of the protons of mass $m$ traveling at velocity $\mathrm{v}$ is transferred to the target material of density $\mathrm{n}$ by electronic excitation in the target. The beam particle energy lost by the projectile to the target per unit path length, or target stopping power [2], is 
related to the stopping cross section of the target, $S(v)$ by:

$$
-\frac{d E}{d x}=n S(v)
$$

The stopping cross section is given by:

$$
S(v)=\frac{4 \pi e^{4} Z_{1}^{2} Z_{2}}{m v^{2}} \ln \frac{2 m v^{2}}{I_{0}}
$$

Here, Z1 and Z2are the projectile charge and the target electron number. I0 is the mean excitation energy of the target material, and it measures the ability of the target to absorb energy from the projectile. I0 can be determined from the dipole oscillator strength distribution (f) of the target:

$$
\ln I_{0}=\frac{\int \frac{d f}{d E} \ln E d E}{\int \frac{d f}{d E} d E}
$$

Using this scheme, the mean excitation energies and thus the stopping powers of atoms and ions can be calculated.

Recently, the mean excitation energies for the second and third row atoms and their ions have been reported [3]. As biological tissue is composed primarily of lower $\mathrm{Z}$ atoms with lower mean excitation energies and higher stopping powers, particle beam irradiation will give large energy deposition into the target, leading to tissue, and thus to tumor, destruction. However, neutral atom mean excitation energies are not the only things of interest. Ions are also of interest.

\begin{tabular}{|c|c|c|c|c|}
\hline Atom & $\mathbf{q = 0}$ & $\mathbf{q = + 2}$ & $\mathbf{q = + 5}$ & $\mathbf{q = + 8}$ \\
\hline $\mathrm{H}$ & 14.99 & -- & -- & -- \\
\hline $\mathrm{He}$ & 42.68 & -- & -- & -- \\
\hline $\mathrm{Li}$ & 33.1 & 134.5 & -- & -- \\
\hline $\mathrm{Be}$ & 42.2 & 205 & -- & -- \\
\hline $\mathrm{B}$ & 52.6 & 136.9 & -- & -- \\
\hline $\mathrm{C}$ & 65.8 & 134.8 & -- & -- \\
\hline $\mathrm{N}$ & 81.6 & 146.4 & 672 & -- \\
\hline $\mathrm{O}$ & 92.9 & 157.2 & 420.7 & -- \\
\hline $\mathrm{F}$ & 116.5 & 176.4 & 370.2 & -- \\
\hline $\mathrm{Ne}$ & 137.2 & 196.9 & 352.6 & 1409.2 \\
\hline $\mathrm{Na}$ & 125.7 & 220.4 & 358.7 & 861.2 \\
\hline $\mathrm{Mg}$ & 128 & 246.8 & 376.7 & 724.8 \\
\hline $\mathrm{Al}$ & 132.2 & 215.8 & 398.8 & 656.4 \\
\hline $\mathrm{Si}$ & 140.8 & 221.2 & 426.5 & 640.1 \\
\hline $\mathrm{P}$ & 151.6 & 225.2 & 458.5 & 648.2 \\
\hline $\mathrm{S}$ & 162.4 & 232.8 & 414.4 & 666.2 \\
\hline $\mathrm{Cl}$ & 174.9 & 242.9 & 395.5 & 694 \\
\hline $\mathrm{Ar}$ & 188.2 & 254 & 394.9 & 728.8 \\
\hline
\end{tabular}

Table 1: Mean Excitation Energies in $\mathrm{eV}$ of the First Three Rows of the Periodic Table and of their $\mathrm{q}=+2$, $\mathrm{q}=+5$, and $\mathrm{q}=+8$ ions

In Table 1, the mean excitation energies of the atoms in the first three rows of the periodic table and of their $\mathrm{q}=+2, \mathrm{q}=+5$, and $\mathrm{q}=+8$ ions are presented [3].
In Figure 2 [4], the mean excitation energies of the unionized atoms of the first three rows are plotted against the atomic number. 


\section{Medicinal \& Analytical Chemistry International Journal}

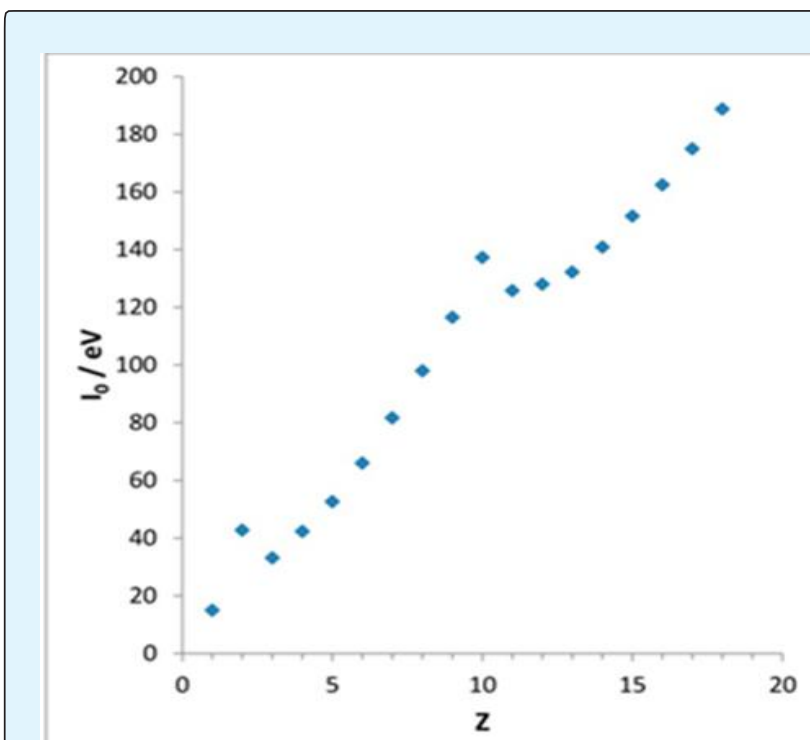

Figure 2: Mean excitation energy (I0) of the atoms in the first three rows of the periodic table as a function of atomic number (Z).

It is clear that $\mathrm{I} 0$ increases with $\mathrm{Z}$ across a row of the table, drops for the beginning of the next row, and then continues, but with a smaller slope then that for the previous row. The slopes (dI0/dZ) for the first three rows from Figure 2 are 15.84, 15.39, and 10.34 respectively.

This behavior is expected [3] from equation 3. For the neutral atoms as expected the energy requirement and the oscillator strength distribution determine the target mean excitation energy, and thus the stopping power. Thus, the biggest changes occur when the electron occupation changes involve occupation changes from one orbital to another. Thus, at this point, rather than increasing with occupation number increase, the mean excitation energy drops significantly. For example from $\mathrm{He}(1 \mathrm{~s} 2)$ to $\mathrm{Li}(1 \mathrm{~s} 22 \mathrm{~s} 1)$ or from $\mathrm{Ne}(\ldots 2 \mathrm{p} 6)$ to $\mathrm{Na}$ (...2p63s1).

Comparing ions of equal charge, for $\mathrm{q}=+2$, the biggest changes occur for $\mathrm{Be} 2+(1 \mathrm{~s} 2)$ to $\mathrm{B} 2+(1 \mathrm{~s} 22 \mathrm{~s} 1)$ and for $\mathrm{Mg} 2+(\ldots 2 \mathrm{p} 6)$ to $\mathrm{Al} 2+(\ldots 2 \mathrm{p} 63 \mathrm{~s} 1)$. For the $\mathrm{q}=+5$ ions, the changes comes from $\mathrm{N} 5+(\ldots 2 \mathrm{~s} 2)$ to $05+(\ldots 2 \mathrm{~s} 22 \mathrm{p} 1)$ and from $\mathrm{P} 5+(2 \mathrm{p} 63 \mathrm{~s} 2)$ to $\mathrm{S} 5+(\ldots 2 \mathrm{p} 63 \mathrm{~s} 23 \mathrm{p} 1)$, and for the $\mathrm{q}=+8$ ions, for $\mathrm{Ne} 8+(1 \mathrm{~s} 2)$ to $\mathrm{Na} 8+(1 \mathrm{~s} 22 \mathrm{~s} 1)$ and from Mg8+ (1s22s2) to Al8+ (1s22s22p1).

Comparing ions of various charges of the same atom, as shown for Ne in Table 2,

\begin{tabular}{|c|c|c|c|c|}
\hline Ion & Occupation & $\mathbf{I}_{\mathbf{0}}(\mathbf{e v})$ & Atom & $\mathbf{I}_{\mathbf{0}}(\mathbf{e v})$ \\
\hline $\mathrm{Ne}$ & $1 \mathrm{~s}^{2} 2 \mathrm{~s}^{2} 2 \mathrm{p}^{6}$ & 137.2 & $\mathrm{Ne}$ & 137.2 \\
\hline $\mathrm{Ne}^{+1}$ & $1 \mathrm{~s}^{2} 2 \mathrm{~s}^{2} 2 \mathrm{p}^{5}$ & 165.2 & $\mathrm{~F}$ & 116.5 \\
\hline $\mathrm{Ne}^{+2}$ & $1 \mathrm{~s}^{2} 2 \mathrm{~s}^{2} 2 \mathrm{p}^{4}$ & 196.9 & $\mathrm{O}$ & 92.9 \\
\hline $\mathrm{Ne}^{+3}$ & $1 \mathrm{~s}^{2} 2 \mathrm{~s}^{2} 2 \mathrm{p}^{3}$ & 235.2 & $\mathrm{~N}$ & 81.6 \\
\hline $\mathrm{Ne}^{+4}$ & $1 \mathrm{~s}^{2} 2 \mathrm{~s}^{2} 2 \mathrm{p}^{2}$ & 282.8 & $\mathrm{C}$ & 65.3 \\
\hline $\mathrm{Ne}^{+5}$ & $1 \mathrm{~s}^{2} 2 \mathrm{~s}^{2} 2 \mathrm{p}^{1}$ & 352.6 & $\mathrm{~B}$ & 52.6 \\
\hline $\mathrm{Ne}^{+6}$ & $1 \mathrm{~s}^{2} 2 \mathrm{~s}^{2}$ & 475 & $\mathrm{Be}$ & 42.2 \\
\hline $\mathrm{Ne}^{+7}$ & $1 \mathrm{~s}^{2} 2 \mathrm{~s}^{1}$ & 696.8 & $\mathrm{Li}$ & 33.1 \\
\hline $\mathrm{Ne}^{+8}$ & $1 \mathrm{~s}^{2}$ & 1409.2 & $\mathrm{He}$ & 42.7 \\
\hline $\mathrm{Ne}^{+9}$ & $1 \mathrm{~s}^{1}$ & 1498.4 & $\mathrm{H}$ & 15 \\
\hline
\end{tabular}

Table 2: Mean Excitation Energies in $\mathrm{eV}$ of $\mathrm{Ne}$ in Various Ionization States and of Atoms with the Same Orbital Occupation [3].

The behavior of the mean excitation energy of these series of systems is governed by the behavior of the dipole oscillator strength distribution for the various systems, and is fully explained in reference 3 . As the stopping power of a system varies inversely as the natural logarithm of the mean excitation energy, the stopping power will decrease with increasing mean excitation energy.

In the case of tumor treatment, individual atoms and monatomic ions are not generally of greatest interest; molecules and functional groups are! Although mean excitation energies of smaller molecules can be calculated directly as described above, for larger systems one usually involves the Bragg Rule [5], and assumes that the stopping cross section of a larger molecule, functional group, or molecular ion can be approximated by an aggregate of fragments and/or functional groups, as in equation 4:

$$
S_{\text {molecule }}(v)=\sum_{i=\text { atoms }} S_{i}(v)
$$

Similarly, the mean excitation energy of the full system would be given as:

$$
\ln I_{0}=\frac{1}{N} \sum_{i=\text { atoms }} n_{i} I_{0}^{i}
$$

The stopping power of such a multi-atom entity can thus be calculated from individual fragment stopping cross sections, or can be calculated as a single entity using eq.5. As the Bragg peak is projectile velocity dependent, 


\section{Medicinal \& Analytical Chemistry International Journal}

the velocity of the projectile beam can be adjusted so that the peak comes at the location of the tumor, and thus the collateral damage is minimized.

\section{References}

1. Sabin JR (2015) Stopping Power of Biological Systems. J Phys Chem Biophys 5(2): e125

2. For a general presentation of the theory of stopping power, see P. Sigmund, Stopping of Heavy Ions - A Theoretical Approach, Springer Tracts in Modern Physics 204(2004).
3. Sauer SPA, Sabin JR, Oddershede J (2018) Zdependence of Mean Excitation Energies for Second and Third Row Atoms and Their Ions. J Chem Phys 148(17): 174307

4. Figure 2 was taken from reference 3.

5. Bragg WH, Kleeman R (1905) On the alpha particles of radium and their loss of range on passing through various atoms and molecules. Philos Mag 10(57): 318.

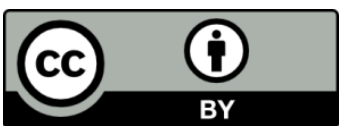

